\title{
Voltage Measurement Errors as a result of Multiple VT Groundings
}

\author{
F. Uriondo ${ }^{1}$, G. Aguirre ${ }^{1}$, J.R. Hernández ${ }^{1}$ and J.M. García ${ }^{2}$ \\ ${ }^{1}$ Department of Electrical Engineering \\ E.S.I. Bilbao, University of the Basque Country \\ Alameda Urquijo s/n - 48013 Bilbao (Spain) \\ Phone/Fax number:+0034 946012 000, e-mail: felipe.uriondo@ehu.es, gabriel.aguirre@ehu.es, \\ joseramon.hernandez@ehu.es, \\ 2 INGETEAM T\&D \\ Pol. Ind. Artunduaga \\ Usasuaga 7 - 48970 Basauri (Spain) \\ Phone/Fax number:+0034 946018 900, e-mail: juanmari.garcia@ingeteam.com
}

\begin{abstract}
On 12 December 2007 a distance relay tripped erroneously by zone 1 for a ground fault in an adjacent line. RTDS simulations and theoretical calculations proved that the relay had operated according to its design parameters and that the settings of the units involved were adequate. Further analysis of the fault recordings showed that the currents followed a single-phase to earth fault pattern, whereas the voltages did not follow any known pattern for the conventional types of faults.
\end{abstract}

In the first part of the paper a theoretical analysis of the problems involved with grounding the VTs in multiple locations is presented. First, the nature of the impedance between different grounding points is studied. Next, the voltage gradient during an earth fault is shown and the sequence voltages affected by a fault involving earth are determined. The deviation from the true value of the faulted phase voltage is also theoretically expounded. The relaying units affected by the multiple groundings are listed and their possible misoperations presented. To end the procedure to determine that such a problem exists and the ways to confirm it are presented along with the calculations that have to be done to determine the performance of the relays involved.

In the second part of the paper the analysis of a real case is presented. The theoretical background expounded in the first part of this work is applied to the above mentioned fault and it is shown that, had only been one VT grounding, the relay would have operated correctly.

\section{Key words}

Voltage measurements, voltage transformers, multiple groundings, distance relaying, directional relaying.

\section{Introduction}

When the VTs are grounded both at the VT location and at the relay location (usually in the relay housing) the situation illustrated in Figure 1 arises.
As it will be shown, the situation can go unnoticed since direct sequence voltages measured in the relay housing are measured correctly in spite of the multiple grounding because the $\Delta \mathrm{V}$ component of the voltages does not affect this measurement. In fact, only the zero sequence voltages measured in the relay housing are affected.

Due to this fact, it is only when zero sequence voltages appear that the problem really manifests. Usually only distance and directional relays use this magnitude and it is their performance which is more affected.

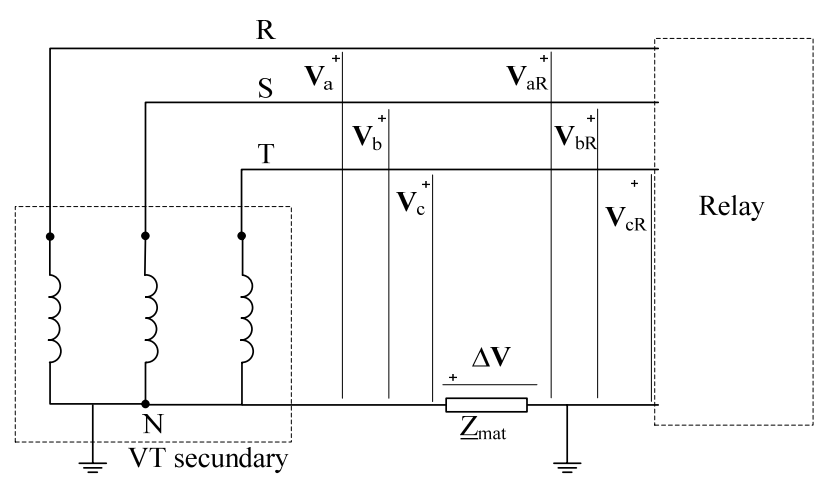

Fig. 1. Groundings in VT and relay locations.

Based on the fact that only the zero sequence voltage is affected, the non existence of the fault in some phases can be checked and the really faulted phases can be determined. This can be achieved with the help of a component orthogonal to the fault that does not use the zero sequence voltage.

Once the faulted phases are determined the term $\Delta \mathrm{V}$ can be estimated using data from the unfaulted phases in a clever manner. In fact, the voltage displacement of the unfaulted phases must be very similar and these 
displacements are used to determine the neutral voltage displacement.

The grounding impedance is capacitive and resistive, but mainly resistive and, using this fact, the reliability of the $\underline{\Delta \mathrm{V}}$ term previously calculated can be independently confirmed. In fact, the $\underline{\Delta V}$ component must be in phase with the zero sequence current due to the mainly resistive nature of the grounding.

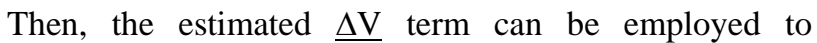
calculate the real fault voltages in the VTs location and use this information to reconstruct the signals that the relay should have received.

Again, with the reconstructed voltages, the validity of the results can be crosschecked using power system data to recalculate the system known parameters. If the system data parameters calculated from the new data are correct, then we can assure that the reconstruction of the voltages has been done correctly. To check this aspect the source impedance is calculated and compared with the expected value and both results must be similar.

\section{Theoretical approach}

A. Nature of the impedance between two grounding points

If we consider two electrodes of any shape grounded in a soil defined by its electric permittivity $\boldsymbol{\varepsilon}$ and electric conductivity $\boldsymbol{\sigma}$ we have the situation presented in Figure 2.

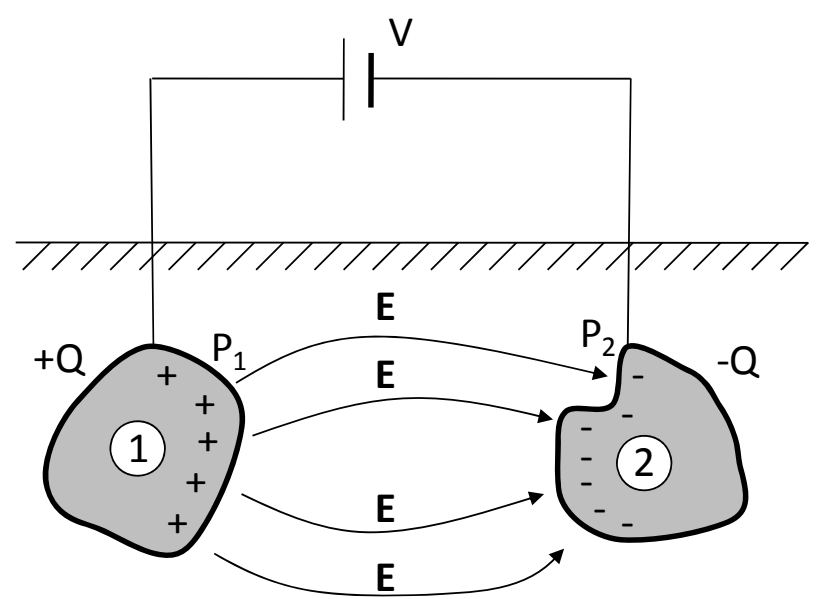

Fig. 2. Two electrodes grounded at different points

\section{1) Capacitance between the electrodes}

In this situation, as can be derived from [1], the total charge in the surface of the electrode 1 can be calculated as:

$$
Q=\oint_{S} \varepsilon \mathbf{E} d S
$$

Where $\mathbf{E}$ is the electric field intensity and $\mathbf{S}$ is the total surface of the electrode.
The voltage between the two electrodes, on the other hand, can be calculated from the following expression:

$$
V=V_{1}-V_{2}=\int_{P_{1}}^{P_{2}} E d l
$$

Where $\mathbf{I}$ is any trajectory between the electrodes 1 and 2 .

Thus, the total capacitance of the system can be derived from its definition:

$$
C=\frac{Q}{V}=\varepsilon \frac{\oint_{S} E d S}{\int_{P_{1}}^{P_{2}} E d l}
$$

\section{2) Resistance between the electrodes}

The total current between the electrodes can be calculated as:

$$
I=\oint_{S} \mathrm{~J} d S=\oint_{S} \sigma \mathrm{E} d S
$$

Taking into account expression (2) to calculate the voltage between the electrodes, the resistance between the electrodes can be calculated as:

$$
R=\frac{V}{I}=\frac{\int_{P_{1}}^{P_{2}} E d \mathbf{l}}{\sigma \oint_{S} E d S}
$$

3) Relation between the resistance and the capacitance

Comparing (3) and (5) we conclude that, between the electrodes, the resistance and capacitance are related by the following expression:

$$
R C=\frac{\varepsilon}{\sigma}
$$

This means that the impedance between electrodes is not purely resistive, as it has a capacitive component. The practical aspect of this fact is that the voltage and current between the electrodes are not in phase, the current is advanced respect to the voltage, as it is in any capacitive charge.

As it will be shown, the voltage lags the currents only a few degrees in practical cases.

\section{B. Ground Voltage profile during an earth fault}

When a ground fault occurs [2] the current flowing through the grounding grid generates a voltage that depends on the fault current magnitude and the total grounding resistance as shown in Figure 3.

As shown in the figure, these ground currents flowing through the grid provoke that the voltages at different points of the substation mat are different. If the VTs are 
grounded at different points $\left(\mathrm{GP}_{1}\right.$ and $\left.\mathrm{GP}_{2}\right)$ of the grid this difference is applied to the associated equipment as a secondary voltage. The voltage difference has been exaggerated in the figure as the difference is of only a few volts compared with the substation grid voltage that can be of several kilovolts.

This voltage difference $(\Delta \mathbf{V})$ can have any sign depending on the grounding points and the fault location. Thus, it can be in phase or in counter-phase with respect the currents taken as positive by the equipment connected to the VTs.

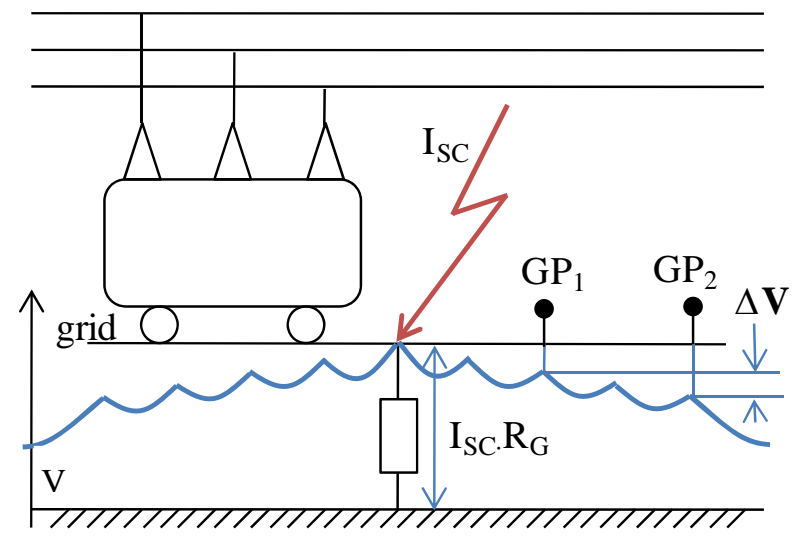

Remote Ground

\section{$\mathrm{I}_{\mathrm{SC}}$ : Short Circuit Current \\ $\mathrm{GP}_{1}$ : Grounding Point 1 \\ $\mathrm{GP}_{2}$ : Grounding Point 2 \\ Grid: Substation Grid}

Fig. 3. Voltage rise during a ground fault

\section{Voltage sequences affected by the multiple grounding}

From Figure 1 we have that the voltages at the relay point $\left(\mathbf{V}_{\mathrm{iR}}\right)$ and the voltages at the $\mathrm{VT}\left(\mathbf{V}_{\mathrm{i}}\right)$ location are related by the following expressions:

$$
\begin{aligned}
& \mathbf{V}_{a R}=\mathbf{V}_{a}+\Delta \mathbf{V} \\
& \mathbf{V}_{b R}=\mathbf{V}_{b}+\Delta \mathbf{V} \\
& \mathbf{V}_{c R}=\mathbf{V}_{c}+\Delta \mathbf{V}
\end{aligned}
$$

If we calculate the direct sequence voltage at the relay point we have that:

$$
\begin{gathered}
\mathbf{V}_{a 1, R}=1 / 3\left(V_{a R}+a \cdot V_{b R}+a^{2} \cdot V_{c R}\right)= \\
=1 / 3\left(V_{a}+a \cdot V_{b}+a^{2} \cdot V_{c}\right)+ \\
+1 / 3\left(1+a+a^{2}\right) \Delta V= \\
=1 / 3\left(V_{a}+a \cdot V_{b}+a^{2} \cdot V_{c}\right)=V_{a 1}
\end{gathered}
$$

So the direct sequence voltage measurement is not affected by the multiple grounding. This is the reason why the problem goes unnoticed until a ground fault happens.

If we calculate the negative sequence voltage at the relay point we have that:

$$
\begin{gathered}
\mathbf{V}_{a 2, R}=1 / 3\left(V_{a R}+a^{2} \cdot V_{b R}+a \cdot V_{c R}\right)= \\
=1 / 3\left(V_{a}+a^{2} \cdot V_{b}+a \cdot V_{c}\right)+ \\
+1 / 3\left(1+a^{2}+a\right) \Delta V= \\
=1 / 3\left(V_{a}+a^{2} \cdot V_{b}+a \cdot V_{c}\right)=V_{a 2}
\end{gathered}
$$

So the negative sequence voltage measurement is not affected by the multiple grounding. This is the reason for which the negative sequence directional units operate correctly even if the problem exists.

As direct and inverse sequence voltages are not affected the problem goes unnoticed also during phase to phase faults.

To end, if we calculate the zero sequence voltage at the relay point, we have that:

$$
\begin{gathered}
V_{a 0, R}=1 / 3\left(V_{a R}+V_{b R}+V_{c R}\right)= \\
=1 / 3\left(V_{a}+V_{b}+V_{c}\right)+ \\
+1 / 3(1+1+1) \Delta V= \\
=1 / 3\left(V_{a}+V_{b}+V_{c}\right)+\Delta V=V_{a 0}+\Delta V
\end{gathered}
$$

So the term $\Delta \mathbf{V}$ affects directly the zero sequence voltage measurement, and only it. Depending on the fault current and the mat impedance between the grounding points this error can be great or can go unnoticed if the impedance between the grounding points is very small.

Due to these reasons, the most affected units are the zero sequence directional units (which use the zero sequence voltage directly) and distance units (which use the phase voltage that is also affected).

\section{Zero sequence voltage and phase voltages deviation}

From (10) we have that:

$$
\mathbf{V}_{a 0, R}=\mathbf{V}_{a 0}+\Delta \mathbf{V}
$$

But the $\Delta \mathbf{V}$ term is created only by the current flowing through earth [3], so it is created only by the zero sequence current since direct and inverse sequences sum zero and do not flow through earth. This zero sequence current creates the voltage drop while circulating through the substation grid, which is mainly resistive. Taking this into account we can rewrite expression (11) as:

$$
\mathbf{V}_{a 0, R}=\mathbf{V}_{a 0} \pm k \cdot \mathbf{I}_{0}
$$

In the expression (11) " $k$ " is a scalar if the grid impedance is purely resistive and complex if the grid impedance has resistive-capacitive nature. In both cases we can consider it a scalar for practical cases.

In Figure 4 the zero sequence voltages at the VT location and at the relay point are shown for a ground fault in the "forward direction". Depending on the $\Delta \mathbf{V}$ magnitude the zero sequence voltage at the relay point can have almost any value and the answer of the zero sequence directional unit can be "forward" or "reverse" depending on the magnitude and direction of the $\Delta \mathbf{V}$ term. 
From (7) we have that:

$$
\begin{aligned}
& \mathbf{V}_{a R}=\mathbf{V}_{a}+\Delta \mathbf{V} \\
& \mathbf{V}_{b R}=\mathbf{V}_{b}+\Delta \mathbf{V} \\
& \mathbf{V}_{c R}=\mathbf{V}_{c}+\Delta \mathbf{V}
\end{aligned}
$$

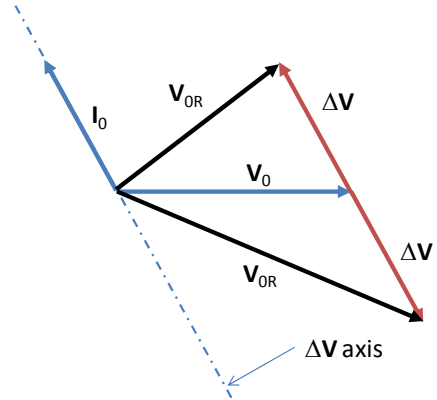

Fig. 4. Zero sequence voltages at the relay point.

Expressing the $\Delta \mathbf{V}$ term as a function of the zero sequence current we have that:

$$
\begin{aligned}
\mathbf{V}_{a R} & =\mathbf{V}_{a} \pm \boldsymbol{k} \cdot \mathbf{I}_{\mathbf{0}} \\
\mathbf{V}_{b R} & =\mathbf{V}_{b} \pm \boldsymbol{k} \cdot \mathbf{I}_{\mathbf{0}} \\
\mathbf{V}_{c R} & =\mathbf{V}_{c} \pm \boldsymbol{k} \cdot \mathbf{I}_{\mathbf{0}}
\end{aligned}
$$

In Figure 5 the phasor diagram for a forward ground fault in the phase "a" is shown.

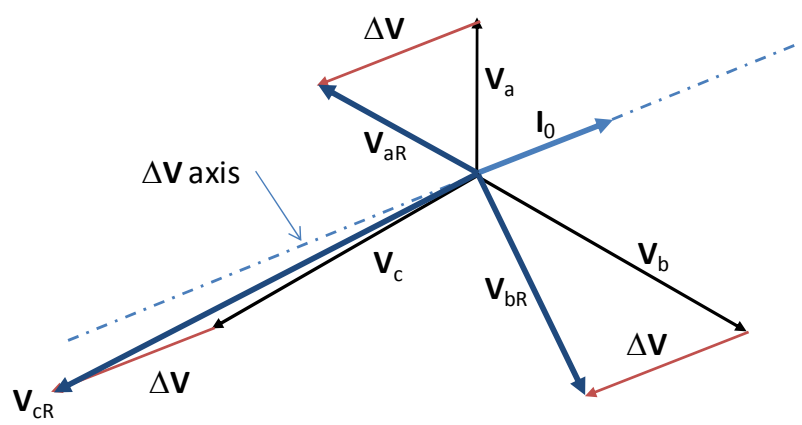

Fig. 5. Phase voltages at the relay point

As it can be seen in the figure, the phase voltages appear completely distorted. The voltage seen by the relay in the faulted phase can be smaller or greater than the real voltage. If the voltage seen by the relay is smaller than the real one, the distance relay is expected to overreach; if it is greater, the relay will probably underreach. In the other two phases, the voltages also appear distorted and the answer of the units related to both phases can vary widely.

E. Performance of the units affected by the voltage distortion

\section{1) Directional unit}

The operating and polarizing signals of the traditional zero sequence directional unit [4] are the following:

$$
\begin{aligned}
& \mathrm{S}_{\text {op }}=\mathrm{I}_{\mathbf{0}} \cdot \mathbf{1} \angle \mathrm{MTA} \\
& \mathrm{S}_{\text {pol }}=-\mathrm{V}_{\mathbf{0}}
\end{aligned}
$$

Where MTA is the maximum torque angle. The condition to be fulfilled by the signals to declare a forward fault is:

$$
\operatorname{Re}\left\{\mathbf{S}_{o p} \cdot \mathbf{S}_{\text {pol }}^{*}\right\}>0
$$

\section{2) Distance units}

A cosine phase comparator is considered to be general enough as to reproduce the performance of the most common distance units employed nowadays.

Some typical operating and polarising signals, used to study the performance of different types of units, are established below:

- Variable ground MHO unit

$$
\begin{aligned}
& \mathbf{S}_{\text {op }}=\left(\mathbf{I}_{\varnothing}-\mathbf{I}_{\mathbf{0}}\right) \mathbf{Z}_{\mathbf{R}}+\mathbf{K}_{\mathbf{0}} \mathbf{Z}_{\mathbf{R}} \mathbf{I}_{\mathbf{0}}-\mathbf{V}_{\emptyset} \\
& \mathbf{S}_{\text {pol }}=\left(\mathbf{V}_{\text {a1 }}\right)_{\text {mem }}
\end{aligned}
$$

- Ground reactance unit

$$
\begin{aligned}
& \mathbf{S}_{o p}=\left(\mathbf{I}_{\emptyset}-\mathbf{I}_{\mathbf{0}}\right) \mathbf{Z}_{\mathbf{R}}+\mathbf{K}_{\mathbf{0}} \mathbf{Z}_{\mathbf{R}} \mathbf{I}_{\mathbf{0}}-\mathbf{V}_{\emptyset} \\
& \mathbf{S}_{\text {pol }}=\mathbf{I}_{\varnothing} \mathbf{Z}_{\mathbf{R}}
\end{aligned}
$$

Where $\mathbf{I}_{\emptyset}$ is the phase current, $\mathbf{Z}_{\mathbf{R}}$ is the line replica impedance and $\mathbf{K}_{\mathbf{0}}$ is:

$$
\mathbf{K}_{\mathbf{0}}=\frac{\mathbf{Z}_{\mathrm{L} 0}}{\mathbf{Z}_{\mathrm{L} 1}}
$$

Being $\mathbf{Z}_{\mathbf{L} \mathbf{0}}, \mathbf{Z}_{\mathbf{L} \mathbf{1}}$ the line zero and direct sequence impedances.

The condition to be fulfilled by the signals to declare a fault inside the protection zone is:

$$
\operatorname{Re}\left\{\mathbf{S}_{\text {op }} \cdot \mathbf{S}_{\text {pol }}^{*}\right\}>0
$$

Inverse and zero sequence reactances can be treated the same way, modifying the value of the polarizing signal using the zero sequence or the inverse sequence current instead of the phase current.

\section{Real case study}

The theoretical analysis previously described is next applied to a real case. We will conclude, on the face of the measurements available, that the relay performed correctly and that it is not the relay manufacturer but the grounding that should be held responsible for its misoperation. As it stands, we will show that had the relay been fed with the correct voltages it would have operated correctly.

The relay, in this case, is located in a very short line so that small measurement errors can easily give rise to a misoperation [4].

\section{A. Prefault and fault data}

In Figure 6 we show the fault as recorded by the distance relay when the fault happened. In Tables I and II are 
presented the prefault and fault phasors calculated using a full cycle cosine filter.

From the data available is straightforward that only one phase current (b-phase) increases its value notably from the prefault to the fault situation and that the three phase voltages vary their value notably.

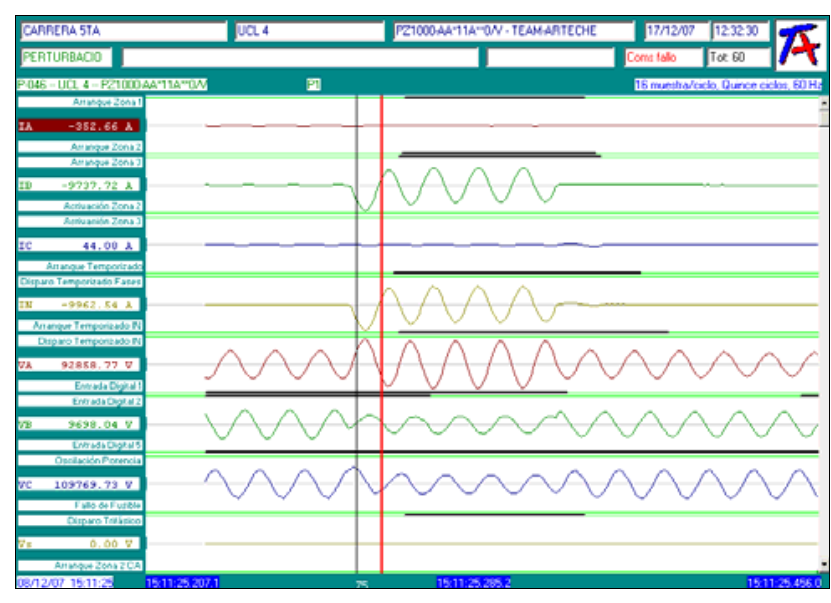

Fig. 6. Fault Data Record.

The variation of the three voltages associated with the variation of only one current can not be associated with any of the possible types of fault (phase to ground, phase to phase, phase-phase to ground or three phase fault).

TABLE I - Prefault phasor values

\begin{tabular}{|c|c|}
\hline Currents & Voltages \\
\hline$I_{a p}=202.3 A \angle 320.9^{\circ}$ & $\mathrm{V}_{a p}=669.662 \mathrm{kV} \angle 0^{\circ}$ \\
\hline$I_{b p}=210.6 A \angle 204.2^{\circ}$ & $\mathrm{V}_{b p}=69.582 \mathrm{kV} \angle 241.8^{\circ}$ \\
\hline$I_{c p}=218.3 \mathrm{~A} \angle 83.2^{\circ}$ & $V_{c p}=68.703 \mathrm{kV} \angle 121.5^{\circ}$ \\
\hline
\end{tabular}

TABLE II - Fault phasor values

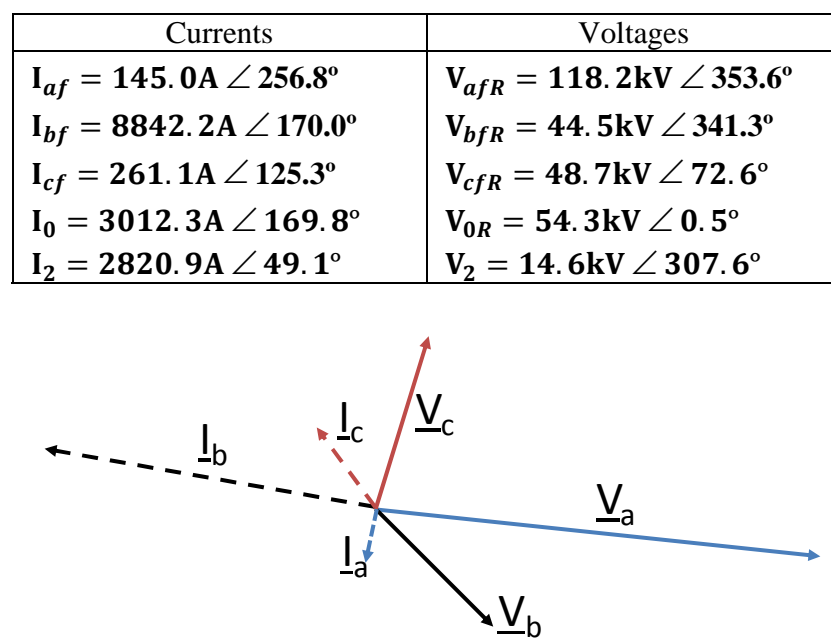

Fig. 7. Fault Phasors.

It can be seen that the currents follow a phase $\mathrm{B}$ to ground fault pattern (the phase $\mathrm{B}$ and neutral currents acquire much higher values than load current) but the voltages show a very awkward response. The A phase voltage almost doubles the prefault value while phase B and $\mathrm{C}$ voltages shrink from normal prefault values to fault voltages. In the case of the "C" phase that reduction in the voltage is not associated with a higher current in that phase. This effect can only take place in a weak infeed situation but the current of phase B clearly shows that it is not the case.

The answer of the zero sequence directional unit calculated using (14) and (15) is that the fault is in the forward direction.

The answer of the zone 1 MHO distance units of the three phases calculated using (16), (18) and (19) is that the fault is into the zone 1 for the phase "b" and out of the zone 1 for phases "a" and "c". But the fact is that the fault happened out of the section covered by the zone 1 MHO distance unit, so it is clearly a misoperation.

\section{B. Discarding fault at " $a$ " and " $c$ " phases}

To discard the possibility that the real fault (not the distorted fault seen by the relay) affects phases "a" and "c" we calculate the $\mathbf{V}_{\text {ac }}$ prefault and fault voltages. If the fault does not affect these phases this voltage should remain unchanged. The reason for this is that:

$$
\mathrm{V}_{a c}=\mathrm{V}_{a c 1}+\mathrm{V}_{a c 2}
$$

So the zero sequence voltage (which is the only one affected by the multiple grounding as shown in the theoretical approach) does not appear in this voltage. Moreover, the "ac" component is orthogonal to the sequences affected by a "b" to ground fault so it must remain unchanged during the fault.

If we calculate these voltages for the actual fault in secondary values we have that:

$$
\begin{array}{ll}
V_{a c p}=V_{a p}-V_{c p} & V_{a c p}=120.7 \mathrm{~V} \angle-29.0^{\circ} \\
V_{a c f R}=V_{a f R}-V_{c f R} & V_{a c f R}=119.0 \mathrm{~V} \angle-30.0^{\circ}
\end{array}
$$

So the $\mathbf{V}_{\mathbf{a c}}$ voltage remains unchanged which is an indicative that the fault does not affect these two phases.

\section{Calculation of the $\Delta \boldsymbol{V}$ term}

In the previous section we have proved that the fault is a "b" to ground fault since phases "a" and "c" are not affected by the fault. In a "b" to ground fault the voltages of the sane phases remain almost unchanged so the variation in the voltages of those two phases is due only to the $\Delta \mathbf{V}$ term introduced by the multiple grounding. Accordingly, the fault voltages at the relay point are the prefault voltages plus the term $\Delta \mathbf{V}$ :

$$
\begin{aligned}
& \mathbf{V}_{a f R}=\mathbf{V}_{a f}+\Delta \mathbf{V}=\mathbf{V}_{a p}+\Delta \mathbf{V} \\
& \mathbf{V}_{c f R}=\mathbf{V}_{c f}+\Delta \mathbf{V}=\mathbf{V}_{c p}+\Delta \mathbf{V}
\end{aligned}
$$

So we can calculate the $\Delta \mathbf{V}$ term for the phases "a" and "c", and both values must coincide:

$$
\begin{aligned}
& \Delta V_{\mathrm{a}}=\mathrm{V}_{a f R}-\mathrm{V}_{a p}=49.6 \mathrm{~V} \angle-15.4^{\circ} \\
& \Delta \mathrm{V}_{\mathrm{c}}=\mathrm{V}_{c f R}-\mathrm{V}_{c p}=51.9 \mathrm{~V} \angle-13.5^{\circ}
\end{aligned}
$$


The small difference between the two values is due mainly to the fact that the fault voltages in phases "a" and "c" differ slightly from the prefault voltages.

\section{Checking of the $\Delta \boldsymbol{V}$ term value}

As shown in the theoretical approach, if the $\Delta \mathbf{V}$ term is correct, this must be in phase or in counter phase with respect to the zero sequence current. This means that the " $k$ " factor (13) must be the same for both phases and almost a scalar. If we do the calculations:

$$
\begin{gathered}
\boldsymbol{k}_{a}=-\frac{\Delta \mathbf{V}_{a}}{\mathbf{I}_{0}} \rightarrow \boldsymbol{k}_{a}=2.77 \angle-6.7^{\circ} \\
\boldsymbol{k}_{c}=-\frac{\Delta \mathbf{V}_{c}}{\mathrm{I}_{0}} \rightarrow \boldsymbol{k}_{c}=2.89 \angle-4.8^{\circ}
\end{gathered}
$$

These results corroborate that the problem is a problem of multiple groundings of the VTs from a third point of view, added to those shown in the two previous sections.

\section{E. Calculation of the real fault voltages}

\section{1) Phase voltages}

We accept that there has been no change in the voltages of the two sane phases ("a" and "c") due to the fault. This means that $\mathbf{V}_{\boldsymbol{a f}}$ and $\mathbf{V}_{\boldsymbol{c f}}$ are equal to $\mathbf{V}_{\boldsymbol{a p}}$ and $\mathbf{V}_{\boldsymbol{c} \boldsymbol{p}}$

We can estimate the $\Delta \mathbf{V}$ term of the faulted phase from the voltage drop of the two sane phases, so:

$$
\Delta \mathbf{V}_{b}=\frac{\Delta \mathbf{V}_{a}+\Delta \mathbf{V}_{c}}{2} \rightarrow \Delta \mathbf{V}_{b}=50.7 \mathrm{~V} \angle-14.4^{\circ}
$$

And the real voltage of the faulted phase is:

$$
V_{b f}=V_{b f R}-\Delta V_{b} \rightarrow V_{b f}=23.4 V \angle-226.9^{\circ}
$$

\section{2) Zero sequence voltage}

We estimate the zero sequence voltage value from the expression:

$$
V_{0}=\frac{V_{a p}+V_{b f}+V_{c p}}{3} \rightarrow V_{0}=14.1 V \angle 68.2^{\circ}
$$

\section{3) Zero sequence voltage checking}

Two independent calculations can be done to check the validity of the zero sequence calculated voltage. The first consists in checking the phase displacement between $\mathbf{V}_{\mathbf{0}}$ and $\mathbf{V}_{\mathbf{2}}$. For a "b" phase to ground fault the displacement must be of about $120^{\circ}$. In our particular case:

$$
\arg \left(V_{0}\right)-\arg \left(V_{2}\right)=120.4^{\circ}
$$

The other one consists in calculating the zero sequence source impedance angle, which must be of about $\mathbf{7 0} \pm \mathbf{1 0}{ }^{\circ}$. In the case studied:

$$
\arg \left(V_{0}\right)+180^{\circ}-\arg \left(I_{0}\right)=76.9^{\circ}
$$

That agrees with the known data of the system parameters.

\section{F. Distance units performance}

The answer of the zone 1 MHO distance units of the three phases calculated using (16), (18) and (19) with the real voltages is that the fault is out of the zone 1 for the three phases. This assures that the misoperation of the "bg” was due to the problem of the multiple grounding and has no relation with incorrect relay operation or wrong relay settings.

\section{Conclusions}

The problem treated in this paper has been cited in different papers ([5],[6]) but in these works the authors simply assert that the VTs must be grounded in a single point, without further explanation. Specifically, they do not discuss the problems that arise when this recommendation is not followed. They neither explain how the incident off-line analysis has to be performed so as to determine what really happened during the fault nor how the existence of the problem can be checked when the multiple groundings are physically near from one another.

In this work a complete overview of the problem is presented. The theoretical aspects of the problem have been detailed and a complete real case incidence analysis that can be used as a guide has been presented.

To end, it must be emphasized that the misoperation of the distance and directional relays when this problem arises can not be solved changing the relay settings or the relay itself, the only way to solve the problem is to eliminate the unnecessary groundings at the relay housing and letting one grounding point for the VTs.

\section{References}

[1] J.F. Mora, Electromagnetismo y Circuitos eléctricos, Mc Graw Hill, Madrid (2005)

[2] Curso Puesta a Tierra Instalaciones Electricas, CIDESPA, Madrid (1993)

[3] P.M. Anderson, Analysis of Faulted Power Systems, IEEE Press, New York (1995)

[4] W. A. Elmore, Protective Relaying Theory and Applications, Marcel Dekker, Inc., New York (1994)

[5] J. Roberts and E. O. Schweitzer, "Limits to the sensitivity of ground directional and distance protection”, 1997 Spring Meeting Of the Pennsylvania Electric Association, Pennsylvania May 15-16, 1997.

[6] K. Zimmerman and J. Mooney, "Comparing ground directional element performance using field data", $20^{\text {th }}$ Annual Western Protective Relay Conference, Spokane, Washington, October 19-21, 1993 